\title{
Effect of dance on lower-limb range of motion in young people with cerebral palsy: a blinded randomized controlled clinical trial
}

This article was published in the following Dove Medical Press journal:

Adolescent Health, Medicine and Therapeutics

\section{Lavinia Teixeira-Machado' Josimari M DeSantana ${ }^{2}$ \\ 'Education in Health Department, Federal University of Sergipe, Lagarto, SE, Brazil; ${ }^{2}$ Department of Physical Therapy, Graduate Program Physiological Sciences, Graduate Program in Health Sciences, Federal University of Sergipe, Aracaju, SE, Brasil}

Purpose: One of the most limiting conditions in cerebral palsy (CP) is the impairment in musculoskeletal mobility. CP may impair the ability to perform efficient movements. The aim of this trial was to investigate the effect of dance on the range of motion (ROM) of lower limbs in young people with CP.

Patients and methods: the randomized clinical trial consisted of two groups: dance group (DG) and control group (CG). Both of them underwent a 1-hour treatment, twice a week, for 2 months. Sanny ${ }^{\mathbb{B}}$ pendulum fleximeter was used to measure ROM in pre- and posttreatment. The applied procedures in both groups were conducted at suitable locations. Twenty-six participants were allocated to these two groups of study.

Results: In DG, the sampling variances showed improvements in all lower limb joints and axes of movements $(P<0.05)$. In $\mathrm{CG}$, there was increase in passive ROM in some lower limb joints $(P \leq 0.05)$.

Conclusion: Physical intervention is imperative to improve ROM. It seems dance practice can contribute to $\mathrm{CP}$ corporal mobility in a positive way.

Clinical trial number: $\mathrm{N}^{\circ}$ CAAE-06154012.4.0000.0058-12, number 98.993.

Keywords: dance, physical therapy, cerebral palsy, range of motion

\section{Introduction}

Cerebral palsy (CP) is a neurological dysfunction that may impair the ability to produce normal levels of range of motion (ROM) and muscular strength. ${ }^{1-3}$ Hypertonicity is a neurological sign of brain damage or motor dysfunction characterized by an increase in tonic stretch reflexes associated with enlarged tendon spasms resulting from hyperexcitability of the stretch reflex. ${ }^{4,5}$ Besides, hypertonia - result of injury to the central nervous system (CNS) - may interfere with patient movement or positioning and then promote pain and discomfort. $^{2}$

This condition also causes other functional impairments such as abnormal postures, excessive activation of antagonistic muscles, and stereotyped movements associated with muscle synergies that difficult various movements as locomotion. ${ }^{3-6}$

Biomechanical impairments are predictors of difficulties in functional balance in several health conditions. The reduced ROM interferes with $\mathrm{CP}$ physical function, and deficits in neuromuscular control increase risk of injuries. ${ }^{7}$ In this view, it is fundamental to assess ROM in CP patients to conduct therapeutic approaches that minimize ROM impairments. Evidence highlights the importance of the goniometry as a method of ROM measurement in hypertonia, as it is performed judiciously by two examiners..$^{7-18}$
Correspondence: Lavinia

Teixeira-Machado

Health Department, Federal University of Sergipe, 13 Marcelo Avenue Lagarto, 49400-000, SE, Brazil

Tel +55793632 2100

Email teixeiramachado@icloud.com 
Approaches focusing on walking training in $\mathrm{CP}$ have been reported. Functional exercises and circuit-training approaches have proven to be beneficial in contributing to and promoting functionality and mobility in $\mathrm{CP}^{19}$ Previous research has elucidated the benefits of rhythmic activities, such as dance, to promote optimizations in motor control and function, because these activities can stimulate responsible systems for interaction of movement, increasing joint mobility and body balance. ${ }^{4,20-25}$

Unfortunately it is unclear how dance intervenes in hypertonia, although some neuroimage studies highlighted the influence of dance in cerebral areas responsible for motor planning, movement control and activating systems, such as the action observation network. In addition, dance practice promotes different effects in white and gray matter. ${ }^{6,27-31}$

Besides, dance practice activates brain connections that influence neuroplasticity by connecting several areas of brain associated with perception, emotion, and motor planning. ${ }^{20,23,24,26-31}$ Some researchers claim neuromuscular actions are intertwined with perception and cognition processes, and the ability to perform tasks is directly related to its interaction with environment, and that determines its functional capability. ${ }^{25,32-36}$

To our knowledge to date, there are limited studies that have investigated dance as a possibility to increase ROM in affected joints by muscle hypertonia. This has led to an interest further investigations into the benefits that dance can have on promoting ROM in CP participants. Thus, the aim of this study was to examine whether dance helps to reduce joint, tendon, and muscle strain, and whether it influences ROM in young people with spastic CP.

\section{Patients and methods}

This study was carried out in accordance with the principles of the Declaration of Helsinki. We declare that this study was approved by the Ethics Committee of Federal University of Sergipe (approval number CAAE-06154012.4.0000.0058-12 and number 98.993) and informed consent form was signed by the participants.

\section{Study design}

This study was a controlled and randomized clinical trial. After signing the informed consent, participants were randomly enrolled into two groups according to a computergenerated randomization sequence: 1) dance group (DG) and 2) control group (CG).

Before the study commenced, the enrolled participants were enrolled into a computer generated system to achieve randomization. A blocking randomization was performed to generate a sequence of allocation to ensure that there was a close balance of the numbers in each group at any time during the study. After every block, the number of participants in each group would be equal, that is, in a 1:1 ratio.

\section{Participants}

Inclusion criteria were as follows: young people with $\mathrm{CP}$; gross motor function classification scale (GMFCS) III-V; both sex; age between 15 and 29 years; muscle hypertonia at the passive movement examination; do not attempt any other physical activity or physical therapy during protocol; do not exhibit cardiopathy or neoplasy. Exclusion criteria were cognitive or psychiatric disturbances and withdraw from study.

Participants were randomly allocated in one of the following groups: DG was composed of young people with $\mathrm{CP}$ who attended dance classes, and CG was composed of participants with $\mathrm{CP}$ who attended physical therapy sessions.

An estimated intended size sample bias to study groups was fixed using the demographics data of the first 12 subjects involved in the study. An $\alpha$ value of 0.05 and a power of $75 \%$ indicated a size sample bias of 12 participants from both groups.

\section{Ethics statement}

All participants' parents signed informed consent for participation in this study and the study was approved by the Ethics Committee of the Federal University of Sergipe.

\section{Experimental protocols Dance group (DG)}

This group received a specific dance protocol (called Técnica Aplicada Lavinia Teixeira [TALT]) based on the concepts of Bartenieff, Feldenkrais, and Laban methods. A total of 24 1-hour sessions were conducted twice a week, for 3 months. Four sets with eight repetitions for each procedure were performed as follows:

1. Global ROM: coordinated and rhythmic dynamic activities in the ROM through floor exercises

2. Motor coordination: coordinated movements of upper and lower limbs emphasizing opposite directions

3. Body image: interaction between practitioner and environment (space -temporal orientation), association of simultaneous components of the movement (temporal coordination), and proprioception (inside knowing/movement perception)

4. Skill and agility: linkage of sequential components of the movement (anticipatory adjustments in the movement 
course) and movements of trunk and head to aid spatial orientation and stimulate equilibrium notions ${ }^{36-38}$

\section{Control group (CG)}

The CG received physical therapeutic exercises, including neurodevelopmental treatment (NDT) and proprioceptive neuromuscular facilitation (PNF) methods, for 1 hour, twice a week, for 3 months, i.e., 24 sessions in total. ${ }^{39-41}$ Four sets with eight repetitions for each procedure were performed:

1. Global ROM with passive and active stretching exercises

2. Motor coordination: coordinated movements of upper and lower limbs emphasizing opposite directions and the proposed diagonals through PNF method

3. Body image: space-temporal orientation, dynamic activities according to NDT

4. Skill and agility: proprioception activities; association of more than one movement to instigate flowing movement with PNF.

\section{Assessment instruments}

\section{Social demographic data}

An enrollment form was used in baseline to collect demographic data (name, age, weight, scholarship, job, marital status, general characters, history of disease, and preceding familiar, physiologic, and social histories) and to record information about functional activities of participants.

\section{Range of motion (ROM)}

Degree of passive ROM was assessed by Sanny ${ }^{\circledR}$ pendular fleximeter. This measurement was performed twice, pre- and posttreatment. Two trained examiners measured the angular measurements at each stage of the study to compare possible variations between the analysis, which was used to determine mean angles among patient groups.
The procedure was repeated in both groups and in both times of sampling. ${ }^{20}$

Movements were assessed bilaterally in the frontal, sagittal, and transversal planes. Hip joint: flexion (flexion of hip with knee in flexion and extension), extension, abduction, adduction, and external and internal rotation; knee joint: flexion and extension movements; ankle joint: dorsal flexion, plantar flexion, abduction, adduction, inversion, and eversion. , $12,22^{2}$

\section{Protocol procedure}

The procedure applied in the DG was performed in a dance class with parallel bars, mirrors, and sound equipment. The procedure applied in the $\mathrm{CG}$ group was performed in a suitable ambulatory for physical therapy (Figure 1).

\section{Statistical analysis}

The Shapiro-Wilk test was used to verify normality. All variables presented normal distribution. Descriptive analysis of demographic data and differences between intra- and intergroups (independent $t$-test) were analyzed using SPSS program, version 22.0 (IBM Corporation, Armonk, NY, USA). Data were represented as mean and standard error of mean. Significance was set at $P \leq 0.05$.

\section{Results}

Thirty-three participants were eligible to participate in the study. Two subjects were excluded from the study because they were $<13$ years of age. Thus, the study began with 31 participants. During the implementation of protocols, two participants concluded physical therapy sessions and other two gave up participating in the study. Twenty-seven participants were distributed into two study groups. Fourteen participants, seven women and seven men, were allocated to physical therapy group (CG). Thirteen participants, seven women and

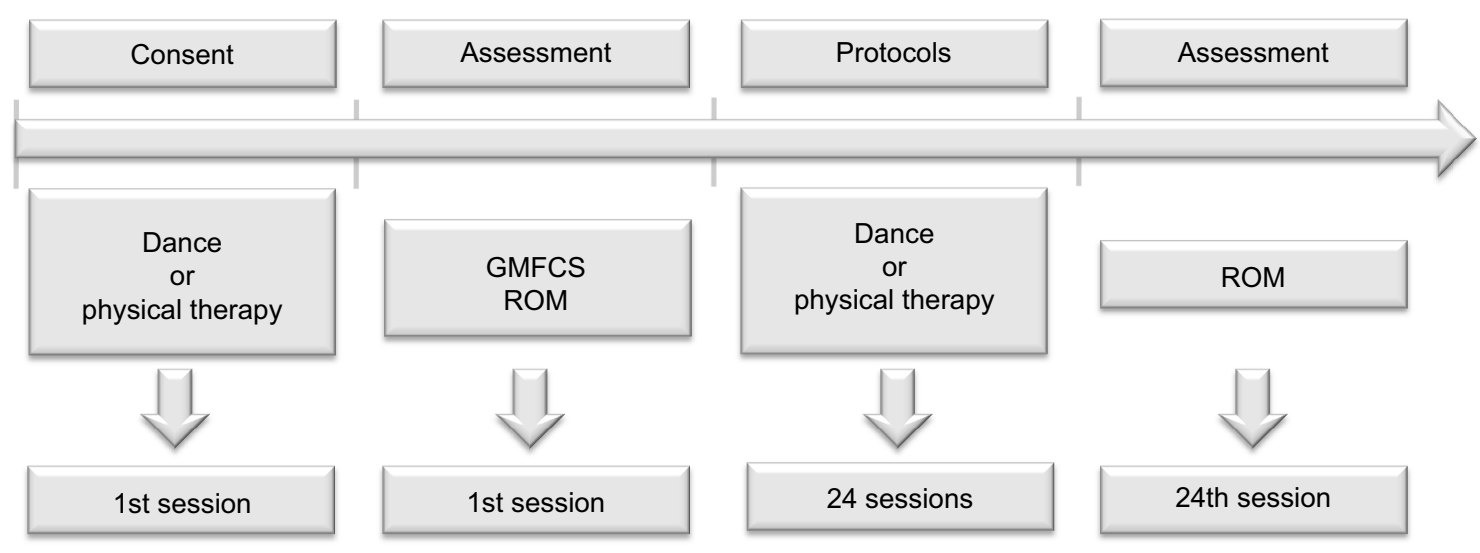

Figure I Timeline of the study.

Abbreviations: GMFCS, gross motor function classification system; ROM, range of motion. 


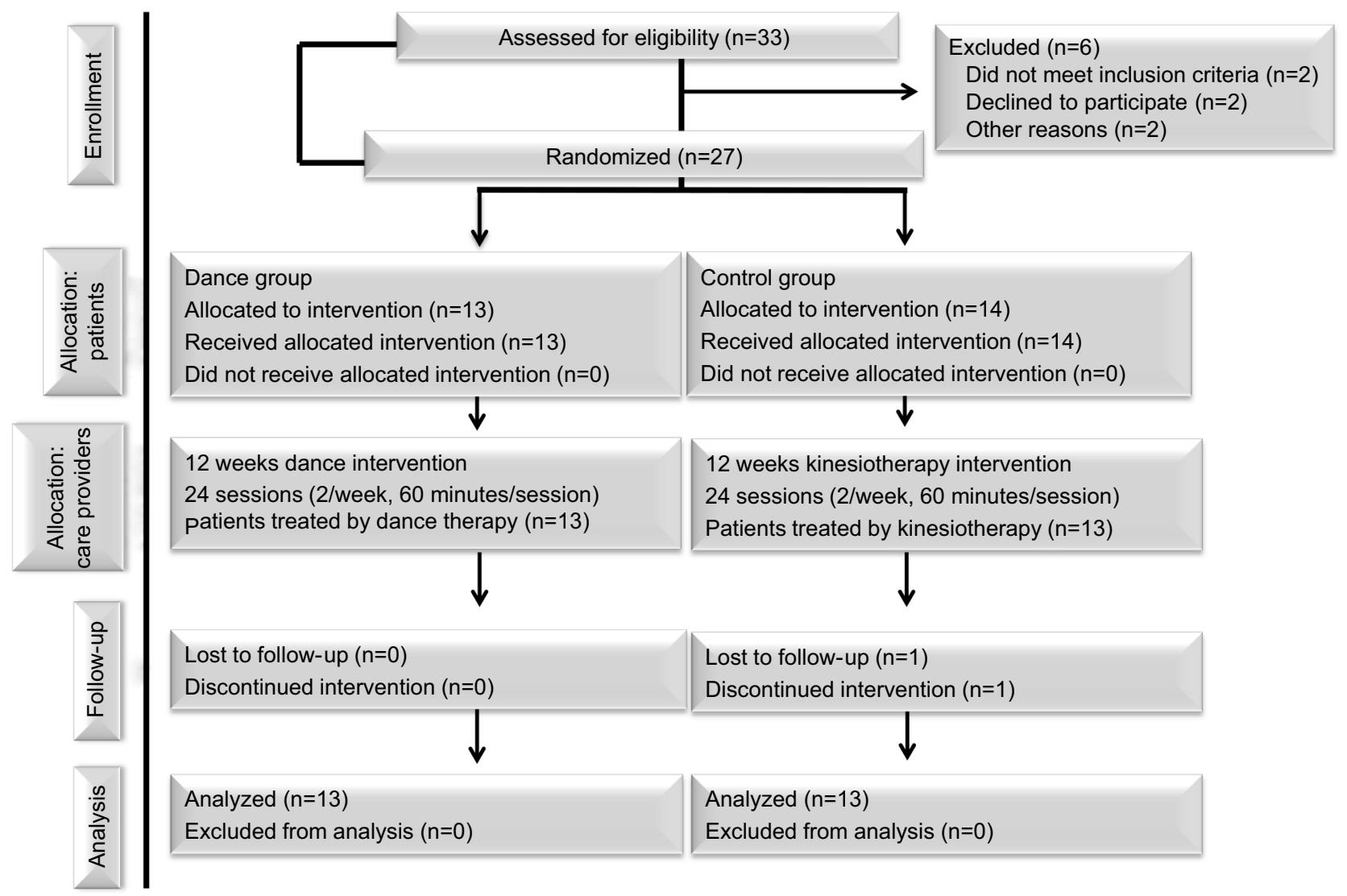

Figure 2 Flow diagram of the study.

six men, were allocated to DG (Figure 2). Sociodemographic data were similar in both groups at baseline (Table 1).

Table 2 describes the movements of the lower limbs of participants in both groups. After treatment, CG showed increase in ROM of the hip for extension ( $P=0.007$, right and $P=0.01$, left), adduction $(P=0.01)$, internal rotation ( $P=0.03$, right and $P=0.002$, left), and left external rotation $(P=0.05)$ and ankle joint, dorsal flexion $(P=0.02$, right and $P=0.03$, left), right plantar flexion $(P=0.01)$, and eversion ( $P=0.0001$, right and $P=0.01$, left improvement adduction on the left hand side and inversion on the right hand side. In DG, compared to baseline, passive ROM in all axes of motion of hip, knee, and ankle joints increased. In the intergroup comparison, DG showed improvement in all axes of motion, except for hip left flexion (with knee extended) $(P=0.16)$ and hip right extension $(P=0.08)$.

\section{Discussion}

Findings showed impairment in all lower limb joints in both groups. For instance, 30 degrees in hip passive flexion
Table I Descriptive and anthropometric characteristics of participants at baseline

\begin{tabular}{|l|l|l|l|}
\hline Baseline data & & & \\
\hline & Dance group & Control group & P-value \\
\hline & $\mathbf{N}=13$ & $\mathbf{N}=14$ & \\
\hline Age (years) & I $4.00(3.46)$ & I5.07 (2.36) & 0.50 \\
\hline Gender & $3 \mathrm{M}$, I0 F & $7 \mathrm{M}, 7 \mathrm{~F}$ & \\
\hline Height $(\mathrm{m})$ & I.38 $(0.13)$ & I.39 $(0.16)$ & 0.78 \\
\hline Weight $(\mathrm{kg})$ & $43.15(\mathrm{I0.7I})$ & $42.18(\mathrm{I0.65})$ & 0.72 \\
\hline BMI $\left(\mathrm{kg} / \mathrm{m}^{2}\right)$ & $22.19(2.02)$ & $21.49(2.57)$ & 0.43 \\
\hline GMFCS & & & \\
\hline Levels & Dance group & Control group & \\
\hline I & - & - & \\
\hline II & - & - & \\
\hline III & 3 & 5 & \\
\hline IV & 3 & 4 & \\
\hline V & 7 & 5 & \\
\hline
\end{tabular}

Notes: GMFCS levels: I, walks without limitations; II, walks with limitations; III, walks using a hand-held mobility device; IV, self-mobility with limitations; may use powered mobility; $\mathrm{V}$, transported in a manual wheelchair. Mean (SD) and $P$-values. $t$-test for independent samples.

Abbreviations: F, female; GMFCS, gross motor function classification system; M, male. 


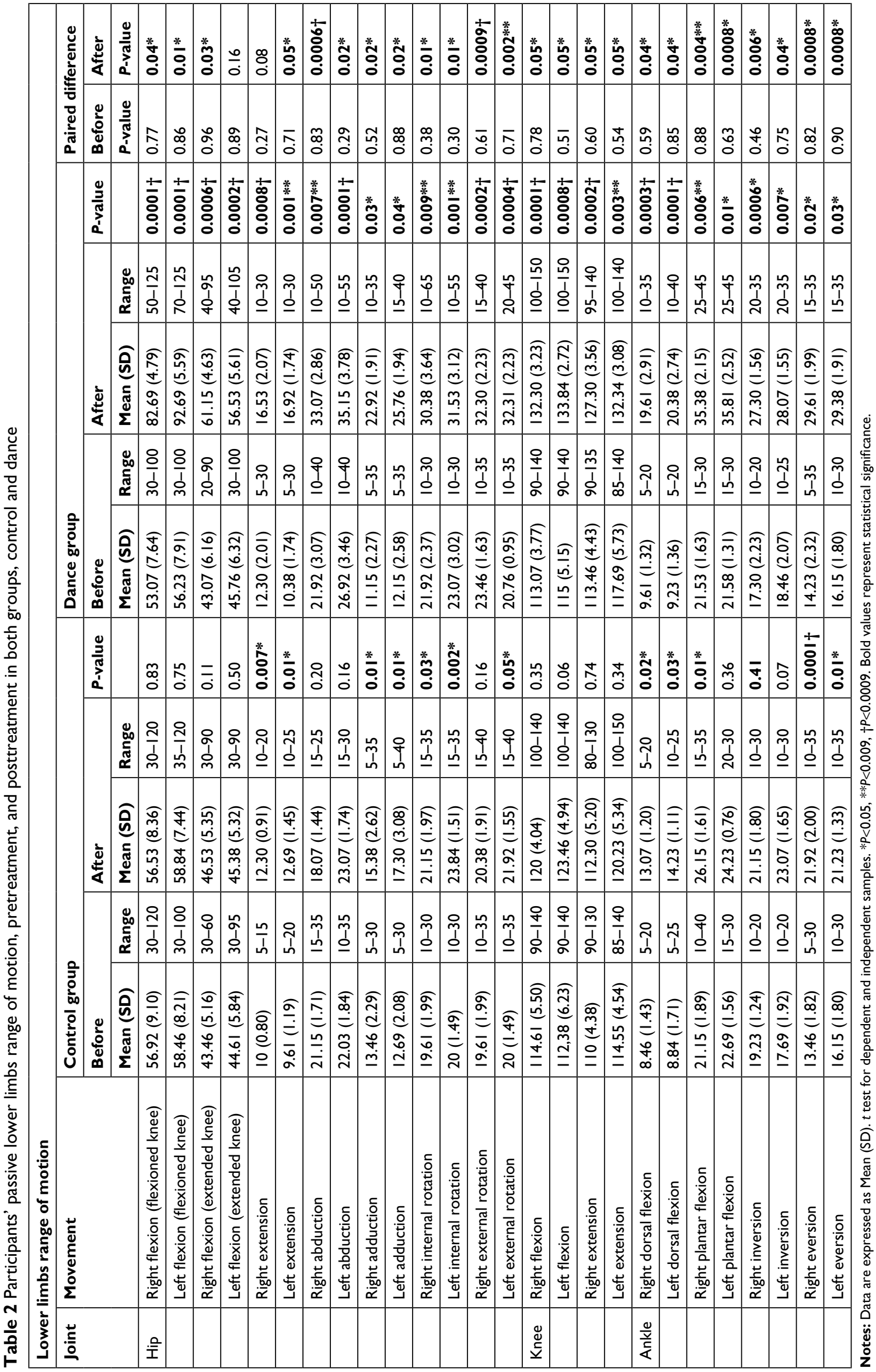


indicates reduced ROM, and consequently, difficulty to perform simple movements, such as transfer changes. Results of this study showed increased ROM in posttreatment. Participants who attended dance classes showed better increase in appendicular ROM.

Dance practice promotes positive effects in special circumstances, including physical disorders. Some studies investigated the influence of dance in joint mobility in various patient groups; Parkinson's disease, ${ }^{3}$ rheumatoid arthritis, ${ }^{42}$ treatment of breast, and lung cancer ${ }^{19}$; and the elderly. ${ }^{24}$

Similar to our findings, several researchers have elucidated the effects of dance on ROM in various disorders. ${ }^{3,10-13,17,20,22,32,35,36,38,42-45}$ Dance involves intentional movements, which supports the findings of our study that it increases ROM of lower limbs, as well as its influence on the movements of head, trunk, and upper limbs were also studied.

Dancing can help increase ROM through movement control, scape, or distraction from pain and stress, change in emotion, state of consciousness, and/or physical ability. Additionally, dance practice can promote health by restoring the bodily system through muscular action and psychological processes, as well as conditioning awareness through movement.

Image studies elucidated the influence of dance practice on cerebral areas that connect motor control and subjective processes. A peculiarity of dance is the possibility of investigating neuronal plasticity and its interaction with behavior. Research suggests that long-term dance practice positively affects brain activity in observation and simulation networks, substantially modifying white and gray matter in various brain regions. Dance integrates several brain functions such as kinesthetic (perception of one's own body movement), musical (interpretation of sound), and emotional (the extent to which music and movement express). ${ }^{25-30}$

Whereas chronic nonprogressive disorders of movement and posture are characterized by a lack of control over the movements that cause muscular weakness and selective loss of motor control, ${ }^{25,36}$ dancing can provide attributes to minimize muscular shortenings dishevel in the corporal biomechanical because of ROM limitation. ${ }^{2,41,46}$ Dance practice protocol used in this study focused on musculoskeletal and movement impairments, highlighting movement potential of each participant.

A range of studies have described the the importance of ROM evaluation in movement disorders and their applicability in the approaches for effectively improving range of motion. Assessing ROM allows a careful investigation of changes in joint mobility and early verification of contractures and deformities, which impair mobility and functional independence.

Participants of our study already presented with many contractures and deformities; we verified ROM deficits and GMFCS levels based on which they were classified. Only a few studies have highlighted these factors in the CP functionality. Researchers usually suggest surgical procedures that compromise the integrity of musculoskeletal structures, in addition to the risks that the patient runs through such interventions.

We had the opportunity to include the participants in a show of a dance school in the theater. This fact had an overwhelming repercussion in the social scope of these participants: being on a stage, in a prominent position, seeing people watching the presentation, perceiving audience enthusiasm with the dance performance, and then receiving standing applause, in addition costumes and stage lighting were among other peculiar characteristics that are awakened in the dance scene were determining factors for the findings. Most of all, we had participants' adherence throughout the study, because the dance program triggered a paramount human need in these young people with $\mathrm{CP}$ : the sense of belonging.

Therefore, we suggest dance practice in $\mathrm{CP}$ cases to promote motor action for inciting favorable levels of ROM. Dancing seems to contribute to body management due to cognitive and motor connection.

\section{Conclusion}

Dance practice can help mobility of young people with CP, and it represents an approach option in kinetic disturbances intervention. Dancing allows the patients to experience communication through the movement of their bodies, and its practice provides individuals to express their feelings, their thoughts and their actions in different ways.

Thus, individuals with neurological and motor disabilities, that present difficulties in expressing their individuality in the face of society, can benefit from dance practice. This is because dancing promotes self-esteem, self-confidence, and well-being, which are factors that are essential for psychomotor performance. This can be further justified by our study findings about increased ROM in all joints in the DG, despite a short period of intervention.

Data from this study suggest future research covering a longer time of intervention to evaluate applicability. New studies are also necessary to investigate the influence of dance practice on joint, tendon, and muscle stress in young people with CP. Studies will also need to evaluate whether 
this intervention alters or maintains skeletal muscle structures in this population.

\section{Data sharing statement}

We do not intend to share individual deidentified participant data. We will share ROM data included in this study. We can share study-related documents, such as photos or videos of participants. When necessary, we can provide our data for further investigations.

\section{Disclosure}

The authors report no conflicts of interest in this work.

\section{References}

1. Mathewson MA, Lieber RL. Pathophysiology of muscle contractures in cerebral palsy. Phys Med Rehabil Clin N Am. 2015;26(1):57-67.

2. Swinnen E, Goten LV, de Koster B, Degelaen M. Thorax and pelvis kinematics during walking, a comparison between children with and without cerebral palsy: a systematic review. NeuroRehabilitation. 2016;38(2):129-146

3. Hackney ME, Earhart GM. Effects of dance on balance and gait in severe Parkinson disease: a case study. Disabil Rehabil. 2010;32(8):679-684

4. Glanzman AM, Swenson AE, Kim H, Heakyun K. Intrarater range of motion reliability in cerebral palsy: a comparison of assessment methods. Pediatr Phys Ther. 2008;20(4):369-372.

5. Champion LM, Chatfield SJ. Measurement of turnout in dance research: a critical review. J Dance Med Sci. 2008;12(4):121-135.

6. Teixeira-Machado L, Azevedo-Santos I, Desantana JM. Dance improves functionality and psychosocial adjustment in cerebral palsy: a randomized controlled clinical trial. Am J Phys Med Rehabil. 2017;96(6):424-429.

7. Lowes LP, Westcott SL, Palisano RJ, Effgen SK, Orlin MN. Muscle force and range of motion as predictors of standing balance in children with cerebral palsy. Phys Occup Ther Pediatr. 2004;24(1-2):57-77.

8. Becker E, Dusing S. Participation is possible: a case report of integration into a community performing arts program. Physiother Theory Pract. 2010;26(4):275-280

9. Allington NJ, Leroy N, Doneux C. Ankle joint range of motion measurements in spastic cerebral palsy children: intraobserver and interobserver reliability and reproducibility of Goniometry and visual estimation. $J$ Pediatr Orthop. 2002;11(3):236-239.

10. Golomer EM, Gravenhorst RM, Toussaint Y. Influence of vision and motor imagery styles on equilibrium control during whole-body rotations. Somatosens Mot Res. 2009;26(4):105-110.

11. Gupta A, Fernihough B, Bailey G, Bombeck P, Clarke A, Hopper D. An evaluation of differences in hip external rotation strength and range of motion between female dancers and non-dancers. Br J Sports Med. 2004;38(6):778-783.

12. Leite MA, Perea DC, Bianchini NM. Effects of active inhibition stretching in hip flexion of ballerinas. Arq Bras Cienc Saúde. 2009;34(3):3-10.

13. Ekstrand J, Wiktorsson M, Oberg B, Gillquist J. Lower extremity goniometric measurements: a study to determine their reliability. Arch Phys Med Rehabil. 1982;63(4):171-175.

14. Robroy LM, McPoil TG. Reliability of ankle Goniometric measurements: a literature review. JAm Pediatr Med Assoc. 2005;95(6):564-572.

15. Lobel EE. The influence of two stretching techniques on standing hip range of motion. J Dance Med Sci. 2016;20(1):38-43.

16. Wu YN, Hwang M, Ren Y, Gaebler-Spira D, Zhang LQ. Combined passive stretching and active movement rehabilitation os lower-limb impairments in children with cerebral palsy using a portable robot Neurorehabil Neural Repair. 2011;25(4):378-385.
17. Wyon M, Felton L, Galloway S. A comparison of two stretching modalities on lower-limb range of motion measurements in recreational dancers. J Strength Cond Res. 2009;23(7):2144-2148.

18. Florêncio LL, Pereira PA, Silva ER, Pegoretti KS, Gonçalves MC, Bevilaqua-Grossi D. Agreement and reliability of two non-invasive methods for assessing cervical range of motion among young adults. Rev Bras Fisioter. 2010;4(2):175-81.

19. Booth ATC, Buizer AI, Meyns P, Oude Lansink ILB, Steenbrink F, van der Krogt MM. The efficacy of functional gait training in children and young adults with cerebral palsy: a systematic review and meta-analysis. Dev Med Child Neurol. 2018;60(9):866-883.

20. Christensen JF, Pollick FE, Lambrechts A, Gomila A, Aktas G, Ogce F. Affective responses to dance. Acta Psychol. 2016;168:91-105.

21. Golomer E, ToussaintY, Bouillette A, Keller J. Spontaneous whole body rotations and classical dance expertise: how shoulder-hip coordination influences supporting leg displacements. J Electromyogr Kinesiol. 2009;19(2):314-321.

22. García-Dantas A, Quested E. The effect of manipulated and accurate assessment feedback on the self-efficacy of dance students. $J$ Dance Med Sci. 2015;19(1):22-30.

23. Ashoori A, Eagleman DM, Jankovic J. Effects of auditory rhythm and music on gait disturbances in Parkinson's disease. Front Neurol. 2015;11(6):234-239.

24. Keller PE, Novembre G, Hove MJ. Rhythm in joint action: psychological and neurophysiological mechanisms for real-time interpersonal coordination. Philos Trans R Soc Lond B: Biol Sci. 2014;369(1658): 20130394

25. Giacosa C, Karpati FJ, Foster NE, Penhune VB, Hyde KL. Dance and music training have different effects on white matter diffusivity in sensorimotor pathways. NeuroImage. 2016;135:273-286.

26. Chauvigné LAS, Belyk M, Brown S. Taking two to tango: fMRI analysis of improvised joint action with physical contact. PLoS One. 2018;13(1):e0191098

27. Bar RJ, Desouza JFX. Tracking plasticity: effects of long-term rehearsal in expert dancers encoding music to movement. PLoS One. 2016;11(1): 0147731 .

28. di Nota PM, Levkov G, Bar R, DeSouza JFX. Lateral occipitotemporal cortex (LOTC) activity is greatest while viewing dance compared to visualization and movement: learning and expertise effects. Exp Brain Res. 2016;234(7):2007-2023.

29. Kirsch LP, Dawson K, Cross ES. Dance experience sculpts aesthetic perception and related brain circuits. Ann N Y Acad Sci. 2015;1337(1): 130-139.

30. Rehfeld K, Müller P, Aye N, et al. Dancing or fitness sport? The effects of two training programs on hippocampal plasticity and balance abilities in healthy seniors. Front. Hum. Neurosci. 2017;11:305.

31. Demarin V, Bedeković MR, Puretić MB, Pašić MB. Arts, brain and cognition. Psychiatr Danub. 2016;28(4):343-348.

32. Veronese N, Maggi S, Schofield P, Stubbs B. Dance movement therapy and falls prevention. Maturitas. 2017;102:1-5.

33. Takakusaki K. Functional neuroanatomy for posture and gait control. J Mov Disorders. 2017;10(1):1-17.

34. Moffet H, Noreau L, Parent É, Drolet M. Feasibility of an eight-week dance-based exercise program and its effects on locomotor ability of persons with functional class III rheumatoid arthritis. Arthritis Care Res. 2000;13(2):100-111.

35. Rodrigues-Krause J, Farinha JB, Krause M, Reischak-Oliveira Á. Effects of dance interventions on cardiovascular risk with ageing: systematic review and meta-analysis. Complement Ther Med. 2016;29:16-28.

36. Madden JR, Mowry P, Gao D, Cullen PM, Foreman NK. Creative arts therapy improves quality of life for pediatric brain tumor patients receiving outpatient chemotherapy. J Pediatr Oncol Nurs. 2010;27(3): 133-145.

37. Teixeira-Machado L, Araujo FM, Cunha FA, Menezes M, Menezes M, Desantana JM. Feldenkrais method-based exercise improves quality of life in individuals with Parkinson's disease: a controlled, randomized clinical trial. Altern Ther Health Med. 2015;21(1):8-14. 
38. Kim SJ, Kwak EE, Park ES, Cho S-R. Differential effects of rhythmic auditory stimulation and neurodevelopmental treatment/Bobath on gait patterns in adults with cerebral palsy: a randomized controlled trial. Clin Rehabil. 2012;26(10):904-914.

39. Grazziotin dos Santos C, Pagnussat AS, Simon AS, Py R, Pinho AS, Wagner MB. Humeral external rotation handling by using the Bobath concept approach affects trunk extensor muscles electromyography in children with cerebral palsy. Res Dev Disabil. 2015;36C:134-141.

40. Li JX, Xu DQ, Hoshizaki B. Proprioception of foot and ankle complex in young regular practitioners of ice hockey, ballet dancing and running. Res Sports Med. 2009;17(4):206-216.

41. Apostolopoulos N, Metsios GS, Flouris AD, Koutedakis Y, Wyon MA. The relevance of stretch intensity and position-a systematic review. Front Psychol. 2015;6:1128.

42. Chang M, Halaki M, Adams R, Cobley S, Lee KY, O’Dwyer N. An exploration of the perception of dance and its relation to biomechanical motion: a systematic review and narrative synthesis. J Dance Med Sci. 2016;20(3):127-136.
43. Sandel SL, Judge JO, Landry N, Faria L, Ouellette R, Majczak M. Dance and movement program improves quality-of-life measures in breast cancer survivors. Cancer Nursing. 2005;28(4):301-309.

44. Kostic R, Uzunovic S, Purenovic-Ivanovic T, et al. The effects of dance training program on the postural stability of middle age women. Cent Eur J Public Health. 2015;S:67-73.

45. Telfer S, Lange MJ, Sudduth ASM. Factors influencing knee adduction moment measurement: a systematic review and meta-regression analysis. Gait Posture. 2017;58:333-339.

46. Yoo JW, Lee DR, Cha YJ, You SH. Augmented effects of EMG biofeedback interfaced with virtual reality on neuromuscular control and movement coordination during reaching in children with cerebral palsy. NeuroRehabilitation. 2017;40(2):175-185.
Adolescent Health, Medicine and Therapeutics

\section{Publish your work in this journal}

Adolescent Health, Medicine and Therapeutics is an international, peer-reviewed, open access journal focusing on health, pathology, and treatment issues specific to the adolescent age group. All aspects of health maintenance, preventative measures and disease treatment interventions are addressed within the journal and practitioners from all disciplines are

Submit your manuscript here: http://www.dovepress.com/adolescent-health-medicine-and-therapeutics-journal

invited to submit their work as well as healthcare researchers and patient support groups. This journal is included in PubMed. The manuscript management system is completely online and includes a very quick and fair peer-review system. Visit http://www.dovepress.com/testimonials. php to read real quotes from published authors. 